\title{
5G TECHNOLOGY FOR AUGMENTED AND VIRTUAL REALITY IN EDUCATION
}

\author{
Adriano Baratè, Goffredo Haus, Luca A. Ludovico, Elena Pagani, \& Nello Scarabottolo \\ Dipartimento di Informatica "Giovanni Degli Antoni", Università degli Studi di Milano (Italy)
}

\begin{abstract}
This paper deals with the adoption of $5 \mathrm{G}$ technologies in an educational context, focusing on activities based on Augmented Reality (AR) and Virtual Reality (VR). After introducing some scenarios using AR/VR approaches, we will describe the main characteristics of 5G and will provide an example of application in the field of music education.
\end{abstract}

Keywords: 5G, education, augmented reality, virtual reality, cloud infrastructures.

\section{Introduction}

In this paper, we will investigate the possibilities offered by $5 \mathrm{G}$ technology when applied to an educational context, covering different school grades, from primary school to higher education. With respect to current networking technologies, $5 \mathrm{G}$ introduces significant improvements in terms of a larger bandwidth, a more reliable service, very low latencies, and a higher density of devices. These features enable a number of innovative educational services with high-bandwidth and low-latency requirements.

In particular, we will focus on the application of augmented and virtual reality approaches to education, and specifically to lab activities.

Augmented reality (AR) allows enriching the environment surrounding learners with additional information. It is of interest in the field of education thanks to its support to knowledge sharing through the annotation of the environment. Furthermore, it allows supporting impaired students, who may integrate their learning experience through appropriate visual, auditory, and haptic interfaces. Virtual reality (VR) allows substituting the real-world environment with a virtual one. It is useful for lab activities where to manipulate objects in a realistic manner, and to conduct training immune from consequences of possible learners' errors. Both AR and VR supply users with immersive experiences, but they require an infrastructure able to provide strong guarantees of high quality $360^{\circ}$ videos, low-latency two-way interactions, precise localization and orientation of the users. Thanks to $5 \mathrm{G}$, these experiences might be enjoyed also, e.g., on personal devices, from remote and in mobility, thus unveiling a number of innovative educational scenarios.

The paper first analyzes the state of the art from a technological point of view, introducing the key features of AR and VR environments. Then, it presents a number of already-available educational experiences based on these technologies, and discusses both their didactic implications and their limits due to current technological constraints. Afterwards, an in-depth analysis of the potentialities of $5 \mathrm{G}$ technologies is conducted, through the study of supplied services and performance achieved by so far deployed trials, and through an investigation about cloud and edge infrastructures leveraged by $5 \mathrm{G}$ to manage content, correlated with the learning modality (in-presence vs. remote vs. in mobility). The study is used to derive the feasibility of AR and VR for both in-presence and remote learning, and to show how network advances can solve the mentioned issues and open new perspectives in education. Finally, the paper introduces some clarifying examples, in particular the application of AR/VR technologies to the field of music education.

\section{Preliminaries on augmented and virtual reality in education}

AR/VR may be applied to a large range of educational scenarios. In order to understand the impact that AR/VR can have on didactical activities, let us mention our previous experience with an undergraduate, three-year bachelor degree on Security of Computer Systems and Networks, offered also online from 2004/2005 on (Milani et al., 2014). Even if the online version proved to be a very successful 
initiative in terms of number of enrolled students, results in exams, and final graduation grades (Scarabottolo, 2018), it suffers a significant limitation: the possibility of organizing synchronous interactive sessions with a student population mainly constituted by already employed people, having difficulties in connecting through a PC during working hours, thus requiring high bandwidth mobile connections. Besides, distance learning can present relevant limitations to hands-on experiments with physical devices. To overcome these issues, it would be useful to be able to reach students through mobile devices and to organizing activities like:

- broadcasting high quality content from the teacher to a population of around 100 students, simultaneously connected and able to interact in real time, also in a virtual environment;

- re-distributing to the above population high-quality materials produced by single students, in order to share and discuss the lab work carried on by each student in response to teacher's stimuli;

- driving the above population into some AR and VR immersive experience, such as the virtual access to a huge computer center where the teacher shows how to configure various devices.

As far as the last item is concerned, a virtual network of devices may be accessed by learners in order to configure them, to promptly react to a simulated attack of intruders, or to experiment in compromising them with a virus. Usually, these activities cannot be conducted from remote and with the mentioned high number of students because (i) a sufficient number of devices to allow each student to experiment with them is not available, and (ii) working students do not have at their disposal devices with which to experiment at their homes. VR would allow to overcome these problems, provided that bandwidth and latency requirements are met by the underlying network technology.

Several useful applications of AR/VR to other fields of education can be devised. In the field of History and Archeology, AR can supply additional information about, e.g., the alleged sculptor who created a statue in a museum that learners frame with their devices. VR can provide a 3D reconstruction of tunnels under an ancient Egyptian tomb, which cannot be completely visited by unauthorized persons. In the field of Botany, AR can give a learner the complete classification of a plant s/he photographs, along with images of alternative varieties of the same species. In Chemistry, students can play with 3D models of molecules, and perform without risk virtual experiments with dangerous substances.

So far, these experiences are hard to deploy due to the inability of the network of supplying high bitrates to a high number of students also in mobility, and with low latencies so that the immersive experiences are realistic. As an example, the LTE technology provides a bitrate of at most $150 \mathrm{Mbps}$ in downlink, and with a few seconds of latency in case of high number of users.

\section{5G Technology and AR/VR}

The standard document for 5G technology (3GPP, 2019) has been published in March 2018 by 3GPP and officially approved in the Plenary Meeting in June 2018. 5G technology promises to be able to support a number of both traditional and novel applications, such as device-to-device communication and Internet of Things (IoT). In this work, the focus is on 5G functionalities and performance that may ease the implementation of advanced e-learning services leveraging AR/VR. In this context, it is relevant also the capability of facilitating data sharing through the formation of extemporary classrooms anywhere by user devices. To these purposes, an analysis of existing - mainly European - 5G trials is included in order to assess the feasibility of e-learning platforms leveraging this technology.

As far as services are concerned, 5G includes both an ultra-reliable low-latency communications (URLLC) service, and an enhanced mobile broadband (eMBB) service (3GPP, 2019). URLLC aims at providing latencies no greater than $50 \mathrm{~ms}$ and reliability of more than $99.9 \%$ (Li et al., 2018). Hence, it might fit the requirements of $\mathrm{AR}$ and VR applications; though, it will be able to provide a data rate of up to $10 \mathrm{Mbps}$ only. By contrast, eMBB (5G EVE, 2018) aims at providing ultra-high throughput so as to address the needs of users accessing multimedia content, ranging from real-time video streaming to online gaming with 3D 4K video; in particular, it should provide a minimum guaranteed bitrate to users of 100 Mbps. In (5G EVE 2018), the goal for Media \& Entertainment applications is TV service for mobile users with throughput of 100-200 Mbps (with peaks of up to $250 \mathrm{Mbps}$ in downlink) and latency lower than $100 \mathrm{~ms}$. Hence, eMBB seems best suitable for AR/VR applications.

In order to assess the characteristics of $5 \mathrm{G}$ networks in real or realistic environments, a number of experiments are ongoing. For the Bari-Matera installation in Italy, the 5G-PPP consortium (The 5G Infrastructure Public Private Partnership, 2018) reports an obtained throughput of around 3 Gbps with a latency of about $2 \mathrm{~ms}$ (Fastweb, 2018). In this case, 5G is mixed with the LTE technology (Tim, Fastweb and Huawei, 2018); the migration towards pure 5G is scheduled for mid-2019. 
The European 5G Observatory (European 5G Observatory, 2018) provides data from around 180 trials and experiments. From the data analysis, it seems that the most realistic measures have achieved $700 \mathrm{Mbps}$ to $1 \mathrm{Gbps}$ data rate in download; this test was conducted in Finland in urban area, hence possibly with a reasonable user density. Over all experiments, average data rates of 1 to $4.5 \mathrm{Gbps}$ have been achieved for users' devices, and latencies $<5 \mathrm{~ms}$. Since AR/VR applications require from $100 \mathrm{Mbps}$ to a few Gbps of bitrate and a latency below $5 \mathrm{~ms}$ with a reliability of $99 \%, 5 \mathrm{G}$ performance would satisfactorily support the requirements of AR/VR, thus making it the elective technology to deploy innovative e-learning services such as those discussed in this paper.

The 5G technology presents two other characteristics of interest for education services. First, the $5 \mathrm{G}$ infrastructure will include cloud/edge platforms for purposes of network statistics collection and performance monitoring, radio channel reconfiguration according to application demands, and network management and optimization (Taleb et al., 2017). Cloud computing means moving data from peripheral devices possibly scarce in resources, through Internet, to groups of high-performance computers where processing may be more efficiently performed. Edge computing is a variant of cloud computing where processing is executed in servers on the border of the network, thus reducing network traffic and latency. This characteristic perfectly fits with the needs of AR/VR: on one hand, AR/VR contents are likely of large size, and they may be conveniently stored in a cloud node, rather than on users' devices, from where learners may download them, and where processing is performed guaranteeing the low latency requirements. This solution, jointly with $5 \mathrm{G}$ performance, adequately supports blended learning with learners partly in presence and partly remotely distributed across different locations, making the sharing of learning experiences through cyber-spaces possible. On the other hand, in the case of extemporary classrooms, one may think to use the teacher's device - appropriately equipped with memory and computation resources - as the edge server from which learners may download shared contents, and possibly upload their own data produced during the lesson for immediate sharing. This is related with the second relevant characteristics of 5G, namely, its multi-RAT (multi-Radio Access Technology) nature. This means that $5 \mathrm{G}$ will be able to cooperate with other radio technologies. For instance, WiFi is a license-free technology, and WiFi 802.11ac can reach, in real deployments, a bitrate of up to $200 \mathrm{Mbps}$. Two millimeter-wave radio technologies are envisioned to support multimedia applications in 5G networks, namely 802.11ad and 802.15.3c (Niu et al., 2015). These technologies supply very high bitrates, of the order of 3-5 Gbps, in small cells of $10 \mathrm{~m}$ radius. These technologies may be used for high-quality AR/VR content fruition and sharing for in-presence classrooms, with higher performance than that achievable via the pure $5 \mathrm{G}$ technology only, possibly at the expenses of a few compatible routers to connect more cells and enlarge the covered area. Furthermore, license-free technologies allow saving network traffic permitted from the chosen tariff plan.

\section{Application of AR/VR to music education}

A challenging field of application of AR/VR over $5 \mathrm{G}$ is music education. Music implies the exchange of multimedia information, at least in form of high-quality audio streams, in order to support professional applications; moreover, other data types are relevant in a music education context, including symbolic information (score, metadata, lyrics, etc.) and video streams, that are demanding in terms of bandwidth and latency requirements (Baratè et al., 2019b). All the mentioned data should be supported in order to provide a comprehensive environment for distance learning and distributed music performance.

Music education over the net intrinsically poses a number of constraints to network characteristics, and, on top of that, AR/VR approaches add other information layers (Baratè et al., 2018). For this reason, currently available technologies are not suitable for experiencing this kind of didactic activities. In the following, we will discuss some significant case studies that can take benefit from the adoption of $5 \mathrm{G}$ technologies.

A first application is enhancing musical or theatrical live shows through AR techniques to let participants investigate specific aspects of the representation. Examples may include labels that highlight the name and role of characters on stage, subtitles for the lyrics, insights about the plot, a score-following function, etc., as shown in Figure 1. If the traditional audience of a show could be distracted by additional information, the introduction of AR into an educational context can conversely improve the efficacy of the didactic experience, by engaging young people, raising their attention and providing user-tailored aiding tools. For instance, a visually impaired student could watch a high-contrast version or enable a sound description of the scene, a dyslexic child could be administered an alternative score with colored notation, and so on. Usually, the information associated to AR is not particularly demanding in terms of bandwidth, unless user's position has to be continuously tracked, processed by a service provider and finally sent back. In the mentioned example of labels over characters, labels are lightweight text information whose position is demanded to the AR visor; in other cases (e.g., when custom score 
representation must be delivered to specific devices), network requirements are more demanding. In general terms, 5G performance is required when the behavior of a user-tailored application cannot be computed on the user's device, which has to get customized contents in the form of high-quality multimedia streams from a server. Network issues are made even worse when: i) multiple streams have to be delivered simultaneously to each user, such as in multi-layer approaches to music representation (Baratè et al., 2019a), ii) multiple users cannot share the same content since the experience is customized, and iii) fruition takes place in a crowded location or in mobility.

\section{Figure 1. An interface that adds contextual and user-tailored information during an opera performance} (Baratè and Ludovico, 2016).

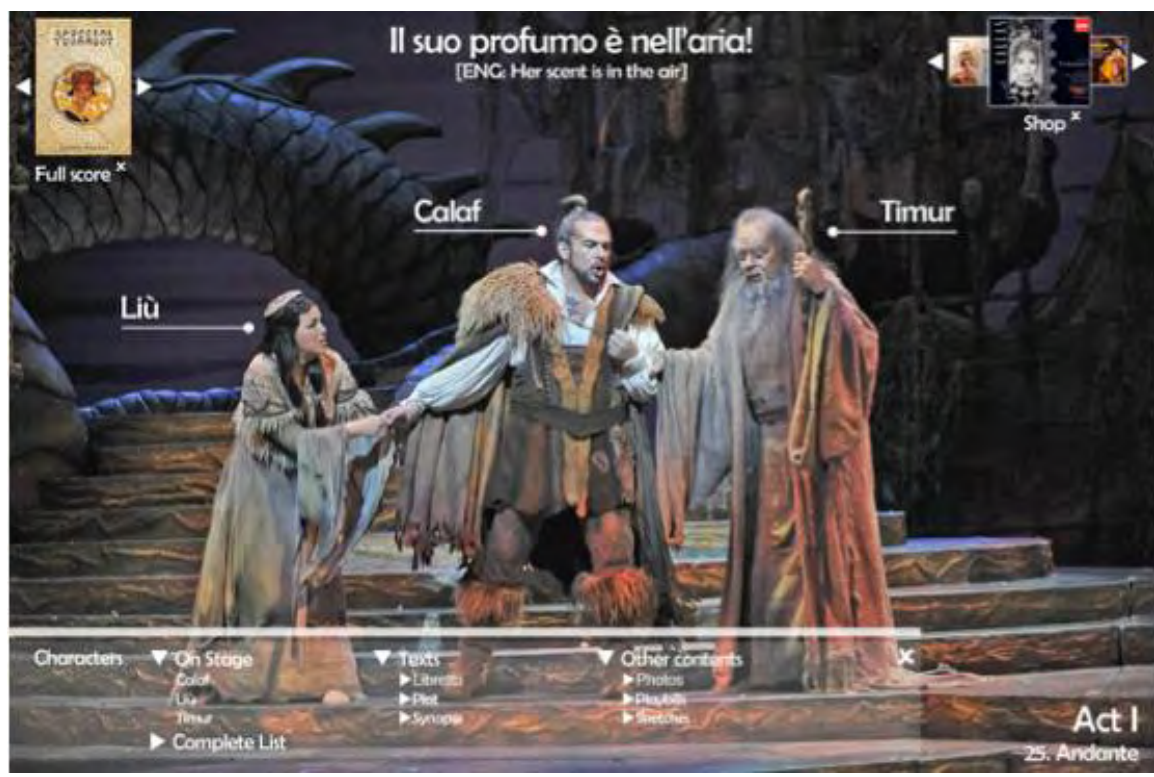

A more advanced use case is the remote participation to live lessons. The idea is to provide online students, who are watching a music performance in real time via the web, with the possibility to rotate their head or even move in the virtual classroom. In this case, user-customized multimedia streams have to reconstruct the remote environment in terms of both high-quality spatialized audio and $4 \mathrm{~K}$ spherical video. This kind of educational application requires also real-time interaction with the teacher and other peers. Once more, the availability of a network technology able to support real-time exchange of multiple media streams with little delay is fundamental, and, with respect to current technologies, only the expected performance of $5 \mathrm{G}$ are apparently suitable for this kind of experience.

In the abovementioned case study, the subject to watch was a real one, e.g., a student playing his/her instrument, captured through a dedicated system such as a 360-degree video camera. Nevertheless, the setting could also be virtual, say an unreal classroom where the teacher and one or many students, geographically distributed worldwide, are able to meet each other and interact virtually. With this kind of VR application, bandwidth and latency requirements are critical.

Finally, in order to explore the third characteristic of $5 \mathrm{G}$, namely the support offered to a high number of devices in a small area, let us mention the case of many network-attached devices interacting in an educational environment to produce a collaborative music performance. For example, the considerable amount of continuous data produced by users' smartphone sensors could be gathered and collectively processed, thus influencing the behavior of a set of virtual instruments as a form of serious game.

Please note that the expected characteristics of $5 \mathrm{G}$ should support all the experiences described so far both in crowded places, like opera houses and concert halls, and in mobility, e.g. when commuting or travelling. These aspects may open up new, unpredictable avenues for future educational activities in music.

\section{Conclusions}

In this paper, we have discussed the possible adoption of $5 \mathrm{G}$ technologies in an educational context, focusing on activities based on Augmented Reality (AR) and Virtual Reality (VR) and providing some examples dealing with the evolution of both online academic courses and music education 
experiences. We have described the expected characteristics and performance of 5G with respect to the requirements of $\mathrm{AR} / \mathrm{VR}$ applications. Current network technologies prove to be unable to fulfil those requirements, while $5 \mathrm{G}$ will provide services that are expected to fit them, thus opening new perspectives in the deployment of innovative educational scenarios.

The real pervasive deployment of $5 \mathrm{G}$ will be able to show the effective performance of the technology, that may significantly differ from those extracted from currently existing trials, due to either high concurrency amongst users in the same cell or to the behavior of future hardware and software components.

\section{References}

3GPP (Feb.2019). Release 15, TR 21.915 v0.6.0. Retrieved Feb. 28, 2019 from http://www.3gpp.org/release-15

5G EVE (2018). 5G European Validation platform for Extensive trials. Retrieved Feb. 28, 2019 from https://www.5g-eve.eu/

Baratè, A., Ludovico, L.A. (2016). Local and Global Semantic Networks for the Representation of Music Information. Journal of e-Learning and Knowledge Society 12 (4), pp.109-123.

Baratè, A., Haus, G., Ludovico, L.A. (2018). Advanced Experience of Music through 5G Technology. Florence Heri-Tech - The Future of Heritage Science and Technologies, IOP Conference Series: Materials Science and Engineering 364, IOP, pp.012021.1-012021.13.

Baratè, A., Haus, G., Ludovico, L.A. (2019a). State of the Art and Perspectives in Multi-Layer Formats for Music Representation. Proc. 2019 Int. Workshop on Multilayer Music Representation and Processing (MMRP 2019), IEEE CPS, pp.27-34.

Baratè, A., Haus, G., Ludovico, L.A., Pagani, E., Scarabottolo, N. (2019b). 5G Technology and Its Applications to Music Education. Proc. 13 $3^{\text {th }}$ Int. Conf. on on e-Learning 2019 (EL 2019), in press.

European 5G Observatory (2018). Major European 5G trials and pilots. Retrieved Feb. 28, 2019 from http://5gobservatory.eu/5g-trial/major-european-5g-trials-and-pilots/

Fastweb (Mar. 2018). Rete 5G a Matera, accesa la prima antenna del progetto Bari-Matera 5G. Retrieved Feb. 28, 2019 from https://www.fastweb.it/internet/5g-matera-fastweb/

Li, Z., Uusitalo, M.A., Shariatmadari, H., Singh, B. (2018). 5G URLLC: Design Challenges and System Concepts. Proc. 15th International Symposium on Wireless Communication Systems (ISWCS).

Milani, M., Papini, S., Scaccia, D., Scarabottolo, N. (2014). Organisation and management of a complete bachelor degree offered online at the University of Milan for ten years. Proc. 8th International Conference on e-Learning 2014, Lisbon, Portugal, pp.87-94.

Niu, Y., Li, Y., Jin, D., Su L., Vasilakos A.V. (2015). A survey of millimeter wave communications (mmWave) for 5G: opportunities and challenges. Wireless Networks 21 (8), Springer, pp. 2657-2676.

Scarabottolo, N. (2018). Comparison of university students enrolled to a dual-mode degree: in presence and online. Proc. EDULEARN18 Conference, pp.576-582.

Taleb, T., Samdanis, K., Mada, B., Flinck, H., Dutta, S., Sabella, D. (2017). On Multi-Access Edge Computing: A Survey of the Emerging 5G Network Edge Cloud Architecture and Orchestration. IEEE Communications Surveys \& Tutorials 19 (3), pp.1657-1681.

The 5G Infrastructure Public Private Partnership (2018). European 5G trials. Retrieved Feb. 28, 2019 from https://5g-ppp.eu/5g-trials-2/

Tim, Fastweb, and Huawei (2018). Bari Matera 5G web site. Retrieved Feb. 28, 2019 from http://www.barimatera5g.it/ 